\title{
Gestionar Más Allá de los Límites de la Empresa Individual: Una Oportunidad Para Obtener Ventajas Competitivas
}

Rafaela Alfalla-Luque ${ }^{1}$

Carmen Medina-Lopez ${ }^{2}$

\section{Resumen}

Cuando un cliente final adquiere un producto está finalizando un proceso que se inició hace días, meses o años y que comenzó con la obtención de las materias primas. Hasta ese acto de compra toda la secuencia de actividades realizadas y todo el valor añadido al producto han debido ser adecuadamente gestionados. La empresa no debería perder el control de su producto hasta que éste no llega a su destinatario final. Por tanto, limitar la gestión únicamente a lo que ocurre dentro de nuestra empresa implica, muy posiblemente, perder oportunidades de conseguir ventajas competitivas sostenibles. Si tenemos en cuenta el fenómeno de la globalización, el rápido desarrollo de las tecnologías, el incremento de los costes logísticos y el aumento de la competencia, parece claro que, cada vez con mayor intensidad, las organizaciones no compiten entre sí de forma individual, sino como parte de la cadena de suministro en la que están inmersas. Por ello, este trabajo se centra en analizar la gestión de ese flujo de materiales e información, que va desde el proveedor de materia prima hasta el consumidor final, así como sus retornos: la Gestión de la Cadena de Suministro.

\begin{abstract}
When customers purchase products, they are bringing a process to a close that had begun days, months or years before and that had started with the raw materials being procured. Up to the point of purchase the whole sequence of activities and all the value added to the product had had to be properly managed. A firm should not lose control over its product until it reaches its final target. Restricting management only to what occurs within the firm therefore very possibly implies that opportunities for gaining sustainable competitive advantages are lost. When the phenomenon of globalization, the rapid developments in technologies, the rise in logistics costs and increased competition are taken into account, it seems clear that organizations are competing less and less between themselves individually, and much more as parts of the supply chains to which they belong. It is for this reason that this paper focuses on analyzing the management of the downstream flows of materials and information from the raw materials supplier right to the final customer and also the returns upstream: Supply Chain Management.
\end{abstract}

Palabras claves: Gestión de la Cadena de Suministro; Dirección de Operaciones; Estrategia; Integración; Supply Chain Management; Operations Management; Strategy; Integration.

\footnotetext{
1 Autor de contacto en: Universidad de Sevilla, GIDEAO Research Group; F.CC. Económicas y Empresariales; Dpto. Economía Financiera y Dirección de Operaciones; Universidad de Sevilla. Correo electrónico: alfalla@us.es

2 Universidad de Sevilla, GIDEAO Research Group
}

Gestionar más allá de los límites de la empresa individual: Una oportunidad para obtener ventajas competitivas 


\section{INTRODUCCIÓN}

La Dirección de Operaciones (DO) se enfrenta a nuevos retos que ofrecen oportunidades emergentes para la obtención de ventajas competitivas sostenibles para la empresa. Entre los nuevos campos a tratar des-

Volumen I

$\mathbf{N}^{\circ} 2$

2010
Pág 115 taca la Gestión de la Cadena de Suministro (GCS) o Supply Chain Management (SCM), pues encontramos una serie de factores que marcan claramente su importancia. Entre ellos (Lummus y Vokurka, 1999; Krajewski y Ritzman, 2000; Heizer y Render, 2009): a) El crecimiento de la competencia; b) El proceso de globalización, que ha situado a clientes y proveedores en cualquier lugar del mundo; c) El menor nivel de integración vertical de las empresas, que han incrementado su especialización y buscan proveedores que puedan abastecerlas con componentes de alta calidad y bajo precio; d) El reconocimiento de que la maximización del desempeño de un eslabón de la cadena de suministro no garantiza su mejor desempeño a nivel de conjunto; e) El rápido avance tecnológico. En concreto, la GCS puede considerarse como la línea de trabajo con mayor potencial, al igual que anteriormente lo han sido la filosofía Justo a Tiempo y el enfoque de la Calidad Total.

La GCS demanda un enfoque de gestión interfuncional, pues implica la coordinación de las funciones básicas de la empresa. Igualmente, traspasa los límites de la empresa individual, llevando a cabo actuaciones encaminadas a una mejor coordinación del flujo de materiales y de información con el resto de empresas implicadas, es decir, con proveedores y clientes, en busca de una adaptación dinámica a las exigencias del mercado. Un porcentaje cada día más elevado del valor añadido a un bien tiene lugar fuera de los límites de la empresa individual (Halldorsson et al., 2007). Por ejemplo, en el sector manufacturero, los artículos y servicios comprados constituyen el 60-70\% del coste de los bienes vendidos (Chase et al., 2000). Por tanto, la administración correcta del flujo de materiales es decisiva. Una adecuada GCS debe orientarse, entre otros, a una racionalización de la base total de proveedores de la compañía, una reducción general de los inventarios de la cadena de suministro, una reducción de los tiempos de espera de la cadena, un recorte de los costes de obsolescencia de la cadena, una reducción del tiempo de respuesta al mercado, una respuesta más rápida a los cambios del mercado y una sincronización de la programación entre compañías (Vollmann et al., 2005). Por tanto, su relevancia estratégica como fuente de ventajas competitivas es manifiesta (Christopher, 1992; Fine, 1998). Gestionar el valor a lo largo de la cadena de suministro se ha convertido en algo crítico para la supervivencia y crecimiento de las compañías.

Sin embargo, a pesar de su clara importancia para la empresa actual, la GCS es todavía un tema que, como analizaremos a lo largo del trabajo, necesita de investigación, aplicación efectiva en el ámbito empresarial y formación en los niveles académicos. Resulta, por tanto, un campo de especial trascendencia para la investigación y a él cual vamos a dedicar el presente trabajo. 


\section{OBJETIVOS Y METODOLOGÍA}

La GCS es una disciplina joven e inmersa en una época de gran actividad investigadora que busca definir sus fronteras y crear un cuerpo de conocimiento sólido que sirva al avance empresarial (Gibson et al., 2005). Por tanto, en estos momentos tiene especial interés la realización de estudios que reflexionen sobre este concepto, hagan manifiesta su importancia y analicen el desarrollo de esta disciplina. Por ello, en este trabajo nos planteamos como objetivos: 1- analizar la Gestión de la Cadena de Suministro y su implicación en la estrategia empresarial. 2- Estudiar el desarrollo docente e investigador en este campo.

Se ha realizado una revisión bibliográfica de artículos (a través de la base de datos de $A B I /$ Informs), libros y actas de los principales congresos de la disciplina (EurOMA, POMS, ACEDE,...) con objeto de establecer qué es la GCS y analizar su impacto en la estrategia empresarial, deteniéndonos en un elemento clave como es la integración de la cadena de suministro. Por otra parte, realizaremos un análisis de los estudios bibliométricos encontrados que han buscado establecer el desarrollo de la investigación en DO y en GCS, así como de las investigaciones y libros de texto que nos permitan conocer la situación de la docencia en GCS. Con ello podremos determinar posibles carencias que han de ser mejoradas en este campo de estudio.

El presente trabajo queda estructurado, en lo que resta, en cuatro apartados. En el siguiente apartado se realizará una delimitación terminológica del concepto de GCS. A continuación, nos detendremos en analizar la importancia estratégica de la CS y la necesidad de integrar la CS para la obtención de ventajas competitivas. El quinto apartado se dedica a revisar el desarrollo docente e investigador de esta disciplina. Finalmente, concluiremos con unas consideraciones finales.

\section{SOBRE LA GESTIÓN DE LA CADENA DE SUMINISTRO}

La GCS nace de un proceso evolutivo progresivo de mejora de la gestión empresarial. Así, para su explicación, Stevens (1989) parte de una clásica división funcional de la empresa, en la que las diferentes funciones son gestionadas como compartimentos independientes entre sí. Sin embargo, las empresas tienen la necesidad de mejorar su gestión y buscan un cierto grado de integración entre las funciones cercanas, dando lugar a la agrupación de las diferentes áreas en tres funciones como son gestión de materiales, gestión de la producción y distribución física. No obstante, en esta etapa siguen existiendo barreras entre dichas funciones que deben ser eliminadas en busca de una integración interna que genere una gestión coordinada e interfuncional. Así, la tercera etapa evolutiva supone la eliminación de las barreras empresariales buscando una integración de la firma con sus proveedores y clientes. Con ello se pretende realizar una gestión global y eficiente de la cadena de suministro en su conjunto, de forma que el objetivo a perseguir sea generar valor a lo largo de toda la cadena y obtener ventajas competitivas sostenibles frente a otras cadenas de suministro. Por tanto, en el propio origen de la GCS está implícita su necesaria aportación a la mejora empresarial y a la obtención de ventajas competitivas sostenibles. 
El término GCS es reciente. Originalmente fue introducido por consultores de la Booz Allen Hamilton a principios de los 80 (Oliver y Webber, 1982). Sin embargo, no será hasta finales de los 90 cuando se produce un creciente interés investigador por este tema (Alfalla-Luque y Medina-Lopez, 2009). Con anterioridad, el concepto más afín a GCS era logística. Por ello, como señala Lambert (2001), hay una gran confusión sobre qué implica exactamente la GCS, pues logística se emplea con frecuencia como sinónimo de GCS. En consecuencia, es necesario realizar algunas consideraciones terminológicas.

Volumen I

$\mathbf{N}^{\circ} 2$

2010
En ocasiones, el concepto de logística se ha definido desde una perspectiva amplia y se ha podido identificar con el de Gestión de la Cadena de Suministro (Schroeder, 2000). Otras veces, los directivos y académicos han definido la logística de una forma más limitada, haciendo referencia al transporte entrante (logística entrante) y la distribución saliente (logística saliente). Actualmente, la logística debe considerarse como una parte de la Gestión de la Cadena de Suministro. En este sentido, podemos observar como el reconocido Council of Logistic Management (CLM) pasa a denominarse a principios de 2005 Council of Supply Chain Management Professionals (CSCMP), pues sus actividades habían superado el concepto tradicional de logística que preconiza el propio organismo. En concreto, el CSCMP define la logística como una parte de la cadena de suministro que tiene como objetivo la planificación, implementación y, control eficiente y efectivo del flujo directo e inverso y almacenamiento de bienes, servicios e información relacionada, desde el origen al cliente, y con el fin de satisfacer sus necesidades. En cambio, según dicho organismo, la GCS incluye la planificación y dirección de todas las actividades relativas a aprovisionamientos, transformación y todas las actividades logísticas. En ella se incluye la coordinación y colaboración con los diferentes miembros de la cadena. Por tanto, en esencia, la GCS integra la gestión de los suministros y la demanda tanto dentro de la empresa como entre las empresas integrantes de la cadena.

En su sentido más amplio, una cadena de suministro se refiere a la forma en la que los materiales fluyen a través de diferentes organizaciones, empezando desde las materias primas y finalizando con los productos terminados que se entregan al consumidor final (Gaither y Frazier, 1999). Por tanto, la Gestión de la Cadena de Suministro supone la planificación, ejecución y control del conjunto de actividades relacionadas con el flujo total de materiales (y el flujo de información asociado), que comienza con el aprovisionamiento de materias primas y finaliza con la entrega de productos terminados a los clientes así como con la recuperación del residuo obtenido, para su reintroducción en la cadena de suministro (Miranda et al., 2005). En ella se incluyen tanto las funciones de aprovisionamiento como las de producción y distribución (directa e inversa).

La GCS tiene el propósito de sincronizar las actividades de una empresa con las de sus proveedores, a fin de acoplar el flujo de materiales, servicios e información con la demanda del cliente (Krajewski y Ritzman, 2000). Una exitosa GCS requiere una integración interfuncional de todos los procesos claves de la empresa dentro de la misma y a través de la red de empresas que comprenden la cadena de suministro (Lambert, 2001).

La cadena de suministro puede involucrar a muchas compañías y a un gran flujo de materiales. Una materia prima puede emplearse en muchos productos terminados diferentes, y por diversas empresas. En general, un producto final se fabrica con materias primas diferentes de proveedores distintos. En consecuencia, las empresas no actúan de manera independiente, sino que forman parte de una cadena de suministro cuya estructura tiene dos dimensiones: la horizontal (número de eslabones que la integran) y la vertical (número de miembros que constituyen cada uno de los eslabones). Su mayor o menor éxito dentro de la cadena va a depender de la capacidad para integrar y coordinar su actividad y las relaciones con el resto de los miembros (Díaz et al., 2004). 
Al analizar y gestionar la cadena de suministro, es necesario conocer quiénes son sus integrantes y clasificarlos en función de la posición que ocupan en la misma (suministradores, productores, vendedores,...). Ello nos va a ayudar en la selección de las relaciones más adecuadas a establecer con cada uno de ellos. En este sentido, Lambert et al. (1998) considera que hay que diferenciar dos categorías, como serían, por un lado, los socios primarios, que constituyen unidades estratégicas de negocio que diseñan y/o crean el producto, $y$, por otro lado, los socios secundarios o de apoyo, que son quienes aportan los recursos o el conocimiento.

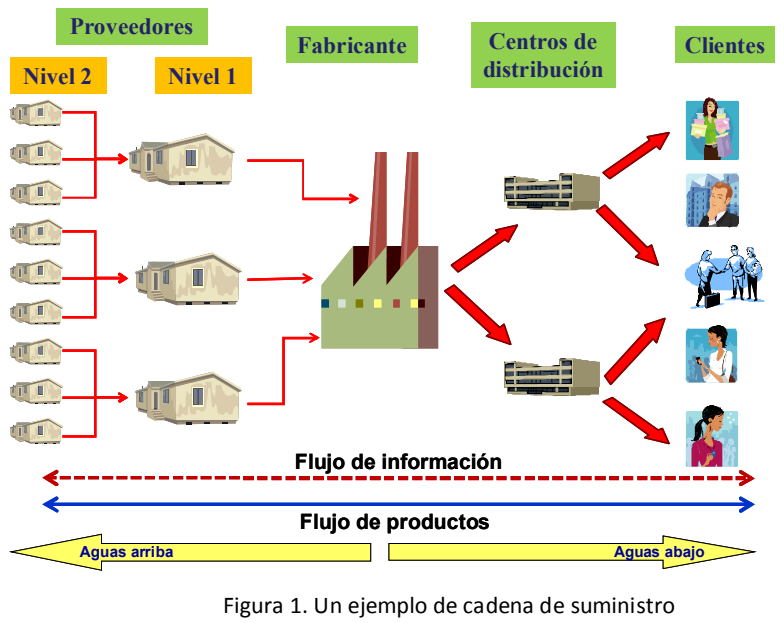

En la Figura 1 se muestra un ejemplo de cadena de suministro en la que se representan cinco eslabones. Respecto a la empresa fabricante encontramos, "aguas arriba", proveedores hasta el segundo nivel (que serán los que aporten las materias primas) y "aguas abajo" aparecen los centros de distribución y los clientes finales. En la cadena de suministro existe un flujo de materiales ascendente que va desde el proveedor de materias primas hasta el cliente final y un flujo descendente que devuelve productos defectuosos, para reciclar, para reutilizar, etc. y que se conoce como logística inversa ${ }^{1}$. Por otra parte, para una adecuada GCS es necesario que fluya también el flujo de información por toda la cadena, tanto en sentido ascendente como descendente.

La GCS incorpora las ventajas de traspasar los límites de la empresa y llevar a cabo actuaciones encaminadas a una mejor coordinación del flujo de materiales y de información con el resto de empresas implicadas en el proceso logístico, es decir, proveedores y clientes, en busca de una adaptación dinámica a las exigencias del mercado (Mejías et al., 2001). No debemos olvidar que el objetivo es construir una cadena de suministro que se enfoque en maximizar el valor para el cliente final (Heizer y Render, 2009).

Gestionar más allá de los límites de la empresa individual: Una oportunidad para obtener ventajas competitivas 


\section{LA IMPORTANCIA ESTRATÉGICA DE GESTIONAR LA CADENA DE SUMINISTRO}

Los directivos actuales conciben que la competencia ya no es sólo empresa a empresa, sino que desborda este plano generándose una competencia cadena de suministro frente a cadena de suministro (Johnson y Pyke, 2000; Christopher, 2000; Ketchen y Guinipero, 2004; Boyer et al., 2005; Ketchen y Hult, 2007). En la actualidad, se ha pasado de un marco competitivo entre empresas a otro en el que son las cadenas de suministro las que compiten, incluyendo éstas tanto la cadena de suministro de los proveedores como la de la empresa compradora y la de los distribuidores comerciales (Christopher, 2000). Por esta razón, es muy importante que las compañías establezcan sus objetivos y estrategias en función de la cadena de suministro a través de la que aportan valor, tanto al producto como al servicio que ofrecen al cliente. En este sentido, se debe buscar la asociación mediante relaciones proveedor-cliente de las empresas que actúan como líderes de la cadena con el resto (que actúan como seguidoras). Así, en cada sector la estrategia de la cadena de suministro será liderada por ciertas organizaciones y apoyada por el resto. No obstante, tanto unas como otras deben comprender y compartir la estrategia de la cadena de suministro de la que forman parte, y orientar sus procesos en función de la misma (Ponce y Prida, 2004).

La GCS tiene claras repercusiones estratégicas. Entre ellas señalaremos, en primer lugar, que sus decisiones apoyan la estrategia empresarial y afectan a diferentes áreas funcionales como Finanzas y Contabilidad, Sistemas de Información o Marketing (Krajewski y Ritzman, 2000; Heizer y Render, 2009). En segundo lugar, mencionar su elevado impacto en costes. El Supply Chain Council, estima que cada 1\% de reducción en el coste de la cadena de suministros equivale a un aumento de los resultados entre el $4 \%$ y el $12 \%$ (Bernabucci, 2008). Es clásica la explicación teórica, defendida por Ballou (1991), de que si los gastos en compras de una empresa representan el $60-80 \%$ de los costes, entonces simplemente con una reducción de precios de compra y servicios del $10 \%$, la empresa puede alcanzar una mejora del $40-50 \%$ en los beneficios antes de impuestos. Esta ecuación corrobora la idea de que los esfuerzos en ventas, para aumentar la facturación, no son tan efectivos en los resultados como la reducción de los costes de la cadena de suministros. En tercer lugar, las decisiones estratégicas adoptadas en GCS (por ejemplo, selección de proveedores, subcontratación de actividades, alianzas estratégicas con proveedores clave, ubicación de fábricas y almacenes, capacidad de producción y almacenaje,...) afectarán la calidad, coste, flexibilidad, servicio, sostenibilidad, riesgo, etc. que definen la obtención de ventajas competitivas. En cuarto lugar, las cadenas de suministro son cada vez más complejas y globales, lo que implica una gestión desde el punto de vista estratégico. Entre las empresas que deben buena parte de su éxito a la Gestión de sus Cadenas de Suministro están Nike, IBM, Walmart, Procter \& Gamble, Xerox, Gillette o Zara (Quinn, 1997).

Porter (1980) fue uno de los primeros autores que identificó la importancia estratégica de la GCS (Figura 2). Así, manifiesta la necesidad de abordar la logística (tanto de entrada ${ }^{2}$ como de salida ${ }^{3}$, las cuales identifica como actividades primarias ${ }^{4}$ ) desde una visión estratégica y de realizar una gestión integrada de toda la cadena de valor, superando el enfoque funcional. En su modelo de fuerzas que mueven la competencia en un sector industrial, Porter (1980) identifica a los eslabones de la cadena de suministro de una organización, es decir, compradores y proveedores, como dos de las cinco fuerzas competitivas básicas (haciendo referencia al poder de negociación de los proveedores y de los compradores). Cualquier empresa, analizada según este modelo y perspectiva, se ve compitiendo por su margen de beneficios "aguas arriba" en la cadena con sus proveedores y "aguas abajo" con sus clientes .

2 Incluye las actividades relacionadas con la recepción, almacenamiento y distribución de los materiales que son adquiridos por la empresa.

3 Relativas a la distribución del producto.

4 A través de las cuales la empresa añade valor a los inputs para sus clientes, quienes, a cambio, están dispuestos a pagar por ello. Son las relacionadas con la creación, venta y transferencia del producto al comprador, así como con la asistencia posventa. 


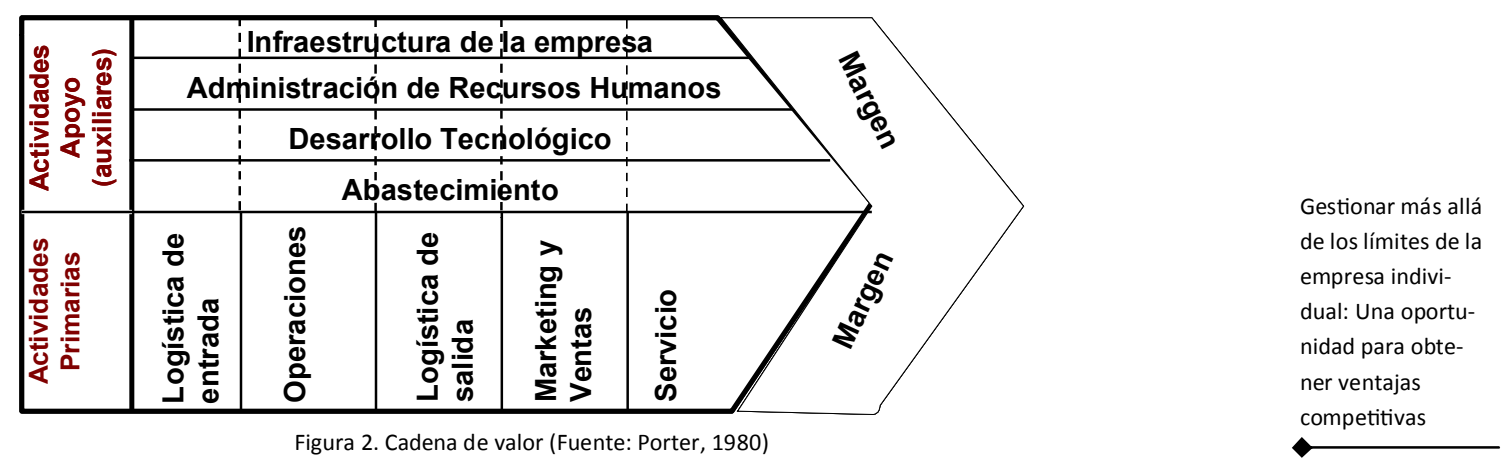

La empresa obtiene una ventaja competitiva en la medida en que desempeña más barato y mejor que sus competidores aquellas actividades de la cadena que son estratégicamente importantes. En términos competitivos, el valor debe ser contemplado desde la perspectiva del cliente final, el cual debe estar dispuesto a pagar una cantidad que sea suficiente para asegurarle a la empresa su margen de beneficio. El margen se concibe como la diferencia existente entre el ingreso y el coste de desempeñar las actividades de valor, que serían tanto las actividades primarias como las de apoyo ${ }^{5}$. Dentro de éstas últimas se incluye el abastecimiento, que engloba todo tipo de compras realizadas por la empresa.

Sin embargo, el valor de la empresa es un eslabón dentro de una cadena de suministro (generadora de valor en su conjunto) que comprende desde los proveedores de las materias primas hasta los consumidores finales. Por tanto, cada vez más, la ventaja competitiva no es provocada por la eficiencia en la gestión de una empresa individual, sino por lo acertadamente que una empresa puede gestionar todo el sistema. Por tanto, el valor que la empresa logre obtener dependerá, en cierta medida, de las relaciones que mantenga con sus clientes y proveedores (Fernández et al., 2003). En consecuencia, debemos analizar la cadena completa de la industria, incluyendo las cadenas de proveedores y clientes.

El análisis de la cadena de valor de una empresa permite identificar sus capacidades, al determinar las actividades en las que ésta posee o puede poseer una ventaja competitiva, así como las relaciones que existan entre ellas. A partir de aquí, la empresa puede identificar las actividades que prefiere realizar por sí misma y aquellas que le conviene adquirir en el exterior (Fernández et al., 2003). Por tanto, partiendo del análisis de esas capacidades empresariales, la organización debe tomar una serie de decisiones estratégicas que van a afectar directamente a la gestión de la cadena de suministro. En concreto, se pueden establecer tres opciones básicas por las que la empresa tiene que decidirse y que van a definir su cadena de suministro: integración vertical, acudir al mercado (subcontratación) o alianzas estratégicas.

Desde la perspectiva estratégica de las decisiones de hacer (integración vertical) o comprar (acudir al mercado) se recomienda a las empresas concentrar sus recursos en el desarrollo de capacidades o competencias esenciales, con las que puedan aportar un valor único a sus clientes y superior al de la competencia. Es preferible subcontratar el resto de actividades periféricas a su actividad básica, en las que la empresa no tiene interés estratégico, ni muestra capacidades específicas que le permitan desarrollarlas en condiciones superiores a la competencia (Ponce y Prida, 2003). 
La integración vertical consiste en la extensión de la empresa hacia cualquiera de los dos extremos del sistema de valor de la industria, a fin de convertirse en su propio suministrador de materiales y/o distribuidor del producto final. Así, una empresa cuya actividad principal sea la fabricación, se puede integrar hacia atrás ("aguas arriba"), es decir, incorporando el aprovisionamiento de los materiales y componentes, o hacia delante ("aguas abajo") interiorizando las actividades de distribución. La empresa tiene que decidir

Volumen I

$\mathbf{N}^{\circ} \mathbf{2}$

2010 qué comprar en el mercado y qué producir internamente e, igualmente, si realiza alguna actividad de distribución o la subcontrata. En la actualidad es difícil realizar una integración vertical total, por lo que se realizan integraciones verticales parciales. En este sentido, las grandes empresas del textil y el calzado están abogando por la integración de las actividades de diseño, compras y marketing de sus productos, si bien una parte importante de sus actividades de fabricación la externalizan a empresas que trabajan en exclusiva para ellas (Fernández et al., 2003). Entre las razones que pueden llevar a optar por la integración vertical podemos destacar (Heizer y Render, 2001):

- Bajar el coste de producción.

- Identificar proveedores inadecuados.

- Asegurar un aprovisionamiento adecuado (cantidad y entrega).

- Utilizar mano de obra o instalaciones excedentes y realizar contribuciones marginales.

- Obtener la calidad deseada.

- Proteger el diseño y la calidad propios.

- Suprimir la colusión de proveedores.

- Obtener un único artículo que pueda suponer un compromiso prohibitivo para un proveedor.

- Conservar a los mejores de la organización y proteger al personal mas capacitado ante reducciones de plantilla.

- Aumentar o mantener el tamaño de la empresa.

Actualmente, la tendencia está orientada hacia la subcontratación, es decir, la búsqueda de proveedores externos de bienes o servicios que anteriormente se proveían dentro de la compañía. La gerencia actual tiende a favorecer la flexibilidad, el enfoque de las fortalezas corporativas, la cercanía al cliente y el mayor énfasis en la productividad y la competencia. Sería raro que una organización fuera superior a la competencia en todos los aspectos de la manufactura o de los servicios. Comprando externamente, la gerencia de la firma puede concentrarse mejor en su misión principal. Esta filosofía ha dado ya como resultado una considerable reducción del tamaño empresarial y una gran amplitud para las compras en el proceso. Teniendo al mundo entero como mercado, la responsabilidad del departamento de compras es buscar o desarrollar proveedores de talla mundial apropiados para satisfacer las necesidades estratégicas de la firma. En estos momentos se subcontrata no sólo la fabricación de productos sino también servicios externos que tradicionalmente se prestaban dentro de la empresa. Dentro de éstos están no sólo la seguridad, los servicios de alimentación y el mantenimiento, sino también la programación informática, la ingeniería, la contabilidad, los servicios jurídicos, la investigación o la logística de los contratos y las compras. De esta manera se ha evolucionado hacia una nueva clase de subcontratación que involucra, en muchas ocasiones, a los servicios (Chase et al., 2000). Lógicamente hay que subcontratar aquellas áreas que no sean clave y esenciales para la empresa. 
En la Tabla 1 se muestran las principales razones que pueden llevar a tomar la decisión estratégica de subcontratar, así como los riesgos que puede llevar implícito dicho proceso. Una adecuada valoración de ambos aspectos aconsejará la subcontratación del producto o servicio o bien optar por otra de las posibles alternativas.

\begin{tabular}{|c|c|}
\hline Razones de la subcontratación & Riesgos de la subcontratación \\
\hline $\begin{array}{l}\text { - Reducción del coste. } \\
\text { - Razones estratégicas. } \\
\text { - Minimización de inventarios, de costes } \\
\text { de movimientos y de otros de los ciclos } \\
\text { de desarrollo y producción. } \\
\text { - Mejora de la eficiencia. } \\
\text { - Adquisición y despliegue de conocimien- } \\
\text { tos periféricos o de tecnología del proce- } \\
\text { so. } \\
\text { - Insuficiente capacidad productiva a cor- } \\
\text { to plazo. } \\
\text { - Escasez o indisponibilidad de los recur- } \\
\text { sos necesarios. } \\
\text { - Razones legales. }\end{array}$ & $\begin{array}{l}\text { - Pérdida de control. } \\
\text { - Barreras de salida más altas. } \\
\text { - Exposición a los riesgos del proveedor. } \\
\text { - Honorarios inesperados a cargos por } \\
\text { - "utilización extra". } \\
\text { - Dificultad para cuantificar las economías. } \\
\text { - Restricciones en la oferta. } \\
\text { - Atención requerida por la alta gerencia. } \\
\text { - Posibilidad de quedar vinculado a una } \\
\text { tecnología obsoleta. } \\
\text { - Preocupaciones con la flexibilidad a largo } \\
\text { plazo y con la satisfacción de los requisi- } \\
\text { tos empresariales cambiantes. }\end{array}$ \\
\hline
\end{tabular}

Gestionar más allá de los límites de la empresa individual: Una oportunidad para obtener ventajas competitivas

Tabla 1. Ventajas e inconvenientes de la subcontratación (Heizer y Render, 2001; Fernández et al., 2003)

En determinadas condiciones establecer acuerdos de cooperación o alianzas estratégicas en fabricación, se presenta como la alternativa más eficiente para el control de las transacciones, superando a la subcontratación y la integración vertical. Una alianza se puede definir como un acuerdo entre dos o más empresas independientes que, uniendo o compartiendo parte de sus capacidades y/o recursos, sin llegar a fusionarse, instauran un cierto grado de interrelación para realizar una o varias actividades que contribuyan a incrementar sus ventajas competitivas. Sin embargo, las empresas mantienen plena autonomía respecto a las actividades que no son objeto de cooperación. Centrándonos en las alianzas en DO, éstas persiguen básicamente economías de escala y de alcance, así como compartir riesgos y conseguir ventajas comparativas de las condiciones locales de un país. También se originan, desde la perspectiva de la inversión internacional, a consecuencia de las presiones del gobierno huésped que obliga a que parte de la fabricación la realicen empresas locales (Fernández et al., 2003). Por tanto, la alianza es eminentemente una actividad económica compartida, encaminada al logro de beneficios mutuos para los participantes. Las empresas que optan por una estrategia basada en este tipo de relación esperan obtener, por un lado, mayor flexibilidad que con una estrategia de integración vertical y, por otro lado, un control sobre las actividades del proveedor muy superior al que podrían obtener si optan por la estrategia basada en la simple negociación en mercado abierto (Ponce y Prida, 2003).

Por tanto, tenemos diferentes opciones estratégicas que van a configurar el diseño de la cadena de suministro y van a condicionar su evolución. Sin embargo, independientemente de la opción elegida, 
siguiendo el enfoque de GCS, nuestra gestión debe ser integral. Es decir, nuestro objetivo debe ser gestionar la cadena de suministro como si de una única empresa se tratase. Para ello es fundamental dirigir nuestras acciones hacia la consecución de una cadena de suministro integrada.

Bagchi et al. (2005) han definido la integración de la CS como la completa colaboración entre la red de miembros de la CS en la toma de decisiones estratégica, táctica y operacional. Esta definición realza la necesidad de que la integración sea efectiva a todos los niveles de la planificación y no estar limitada al nivel operativo.

Las implicaciones estratégicas de una cadena de suministro integrada son claras. Se evidencia que a mayor nivel de integración dentro de la cadena, mayores son los beneficios potenciales que se pueden obtener (Wood 1997; Tan et al. 1998; Frohlich y Westbrook, 2001; Rosenzweig et al., 2003; Cagliano et al., 2004; Kulp et al. 2004; Bagchi et al., 2005). Una cadena de suministro integrada no debe sólo reducir coste, sino que, también, debe crear valor para sus clientes, accionistas y todos los miembros de la cadena. Pero tampoco se debe olvidar que "optimizar" la cadena de suministro significa convencer a los miembros que la integran de que acepten suboptimizaciones locales para el bien del sistema, es decir, buscar la optimización global en vez de la local. La optimización de la cadena de suministro requiere una visión a medio/largo plazo de todos los eslabones que la componen con objeto de mejorar el nivel de servicio al cliente final.

Una integración completa de la cadena de suministro en su conjunto no es una meta fácil de alcanzar. Para conseguirla es necesaria la actuación en un doble frente. En primer lugar, debemos buscar la integración interna. La coordinación entre funciones dentro de la empresa es un prerrequisito crítico para la efectiva integración de la cadena de suministro en su conjunto (Stevens, 1989; Hamblin y Groves, 1995; Forza et al., 2000; Simchi-Levi et al., 2000 Harrison y van Hoek, 2005; Cagliano et al., 2006; Fawcett y Magnan, 2002). Uno de los mayores obstáculos para la completa integración del flujo de materiales y de información a lo largo de la cadena de suministro descansa en un inadecuado sistema interno de dirección de la empresa individual (Romano, 2003; Rosenzweig et al., 2003; Mentzer, 2004). Por tanto, será necesario implicar a las diferentes áreas funcionales.

En segundo lugar, debemos conseguir la integración externa. Ese decir, se han de romper las barreras de la empresa individual y se ha de realizar una integración en ambos sentidos, es decir, tanto hacia atrás (aguas arriba) con proveedores y los proveedores de los proveedores, hasta llegar al proveedor de materia prima, como hacia delante (aguas abajo) con clientes, hasta llegar al cliente final.

En consecuencia, la integración de la cadena de suministro debe romper tanto las barreras entre funciones como entre compañías. Sin embargo, la integración de la cadena de suministro no es un estado absoluto, sino que una cadena puede estar parcialmente integrada -por estar en camino hacia una integración total o por condicionantes internos o externos- (Narasimhan y Kim, 2002; Fawcett y Magnan, 2002; Frohlich y Westbrook, 2001).

Diversos factores han sido establecidos como determinantes en la consecución de una cadena de suministro integrada (Ragatz et al., 1997; Manrodt et al., 1997; Lee, 2000; Mentzer et al., 2001; Frohlich y Westbrook, 2001; Elmuti, 2002; Narasimhan y Kim, 2002; Vickery et al. (2003); Bagchi et al., 2005; Gimenez y Ventura, 2005; Briscoe y Dainty, 2005; Cagliano et al., 2006; van der Vaart y van Donk, 2008, Quesada et al., 2008; Sezen, 2008; Wong y Boon-itt, 2008; Kim, 2009). Estos factores generan actuaciones tanto a nivel estratégico como táctico y operativo. Así, a nivel estratégico, la implicación de la alta dirección en el proceso resulta clave para poder realizar una adecuada integración de la cadena de suministro. En este sentido se deben realizar actuaciones encaminadas hacia: 
- Diseñar adecuadamente la cadena de suministro, definiendo los socios clave que la componen y estableciendo con ellos relaciones a largo plazo.

- Hacer visible la cadena de suministro en la que estamos inmersos, haciendo fluir la información entre funciones y empresas.

- Alinear las estrategias empresariales con los socios clave de la cadena, buscando la misma meta y el mismo enfoque de servicio al cliente.

- Establecer conjuntamente los objetivos a perseguir.

- Compartir ideas, habilidades y cultura organizativa.

- Tener un comportamiento organizacional integrado con los socios de la cadena.

- Realinear incentivos: compartir riesgos, costes y recompensas.

- Definir conjuntamente un sistema de toma de decisiones y de establecimiento de responsabilidades.

- Establecer planes de contingencia conjuntos.

Con el marco establecido en el nivel estratégico, se debe abordar la implantación de una serie de medidas táctico/operativas que hagan posible la integración. Entre ellas destacamos:

- Integración de los sistemas de información, de tal manera que permita comunicarse de forma ágil con los miembros de la cadena de suministro y compartir la información necesaria para el buen funcionamiento de la misma: planes de producción, previsiones de ventas, reabastecimiento, nivel de inventario, pedidos, etc.

- Integración efectiva de los procesos, tanto a nivel interno como a lo largo de la cadena.

- Colaboración, cooperación y toma de decisiones compartida.

- Empleo de equipos logísticos comunes.

- Establecimiento de medidas de rendimiento de la cadena, que permitan evaluar la consecución de los objetivos establecidos a nivel estratégico.

- Establecimiento de equipos de trabajo conjuntos a lo largo de cadena de suministro, que mejoren los procesos y hagan frente a los posibles problemas operativos.

- Definir y mantener los canales de comunicación.

Como puede deducirse del análisis de estos factores, la integración de la cadena no es tarea fácil ni está exenta de dificultades. Algunos estudios ponen de manifiesto que la mayoría de las empresas que practican la integración de la cadena de suministro, limitan su alcance al primer eslabón hacia delante y hacia atrás (Mejza y Wisner, 2001; Fawcett y Magnan, 2002; Bessant et al., 2003). Entre las razones que llevan a un bajo crecimiento de la integración de la cadena de suministro encontramos (Lummus y Vokurka, 1999):

- Incapacidad de alcanzar integración interna en las empresas.

- Falta de una guía para crear alianzas con los socios de la cadena de suministro.

- Fracaso al desarrollar medidas para controlar las alianzas. 
- Falta de compromiso de la alta dirección.

- Incapacidad de extender la visión de la cadena de suministro más allá de compras o distribución para abarcar el conjunto de los procesos empresariales.

Volumen I

$\mathbf{N}^{\circ} 2$

2010

- Falta de confianza dentro y fuera de la compañía.

- Resistencia organizacional al concepto.

- Falta de un sistema integrado de información y comercio electrónico entre las empresas que conforman la cadena de suministro.

Sin embargo, las cadenas de suministro que han tenido la capacidad de vencer estos obstáculos e integrar de forma sólida su cadena (como Dell o Zara) logran obtener una ventaja competitiva sostenible frente a sus rivales. Por tanto, la búsqueda de la integración de los diferentes eslabones que componen la cadena de suministro se convierte en un elemento clave, sobre el que la empresa necesita trabajar para obtener las ventajas de la integración. El reto es conseguir gestionar la cadena de suministro en conjunto, como si de una única empresa se tratase.

\section{DESARROLLO INVESTIGADOR Y DOCENTE DE LA CADENA DE SUMINISTROS}

Puesta de manifiesto la importancia de la GCS tiene para la empresa actual, quedaría conocer si dicha importancia se corresponde con el desarrollo docente e investigador en este campo de estudio. La investigación en GCS es un tema emergente en DO. En este sentido, si analizamos las agendas de investigación realizadas en las últimas décadas, podemos observar la propia evolución de esta área. Cuando Miller y Graham (1981) proponen una agenda de investigación para los 80, ninguno de los temas relacionados con la GCS aparece entre los propuestos, los cuales se centraban en la estrategia de operaciones, la productividad y tecnología, el control de las operaciones y las operaciones en empresas de servicios. Ello se corroboró al analizar la propia evolución de los artículos publicados en las principales revistas de la especialidad (Amoako-Gyampah y Meredith, 1989) ${ }^{6}$, en las que sólo aparecían, al final de la década, escasas publicaciones relativas a compras $(1,66 \%)$ y distribución $(0,28 \%)$. Eran las investigaciones relacionadas con control de inventarios, diseño del proceso y programación de las operaciones las que se situaban claramente destacada en la disciplina. Sin embargo, cuando Amoako-Gyampah y Meredith (1989) analizaron las líneas de investigación en curso, mediante el análisis de las actas de los congresos del Decision Science Institute de los años 1986 y 1987, se pudo observar un aumento de la investigación, tanto en compras como en distribución, en relación con la situación detectada en los artículos analizados, aunque el porcentaje seguía siendo bajo. En concreto, el $6,08 \%$ de los trabajos se centraban en aspectos relativos a compras y el $2,03 \%$ en distribución.

Meredith y Amoako-Gyampah (1990) completan el estudio anterior encuestando a 151 profesores de DO respecto a las líneas de investigación de las tesis doctorales que habían realizado y dirigido. Ello les llevó a un análisis de 308 tesis doctorales. Al realizar una comparativa de la evolución de los temas a lo largo del tiempo, se observaba que los temas más analizados en las tesis doctorales habían sido: programación de operaciones, diseño del proceso y control de inventarios, detectándose un ligero aumento en los últimos años de los temas dedicados a estrategia. Respecto a las líneas de investigación dominantes a principios de los 90, destacaban claramente las cuestiones relativas al diseño del proceso. 
También se produjo un aumento del interés por los temas de estrategia, calidad y operaciones en empresas de servicios. Sin embargo, las cuestiones relacionadas con la cadena de suministro eran objeto de escasas investigaciones: en compras se había desarrollado sólo el $1 \%$ de las tesis analizadas y en distribución el 3,9\%. Además, únicamente el 3,9\% de los investigadores señalaba a compras como una de sus actuales líneas de interés, siendo el porcentaje para distribución todavía menor (2,2\%).

\begin{tabular}{|c|c|c|c|}
\hline Temas & Artículos & Actas de congresos & Variación \\
\hline Calidad & $11.33 \%$ & $27.62 \%$ & $16.29 \%$ \\
\hline Estrategia & $10.82 \%$ & $14.73 \%$ & $3.91 \%$ \\
\hline Programación & $25.93 \%$ & $14.18 \%$ & $-11.75 \%$ \\
\hline Servicios & $2.71 \%$ & $14.00 \%$ & $11.29 \%$ \\
\hline Control de inventarios & $16.18 \%$ & $7.83 \%$ & $-8.35 \%$ \\
\hline Diseño del proceso & $11.28 \%$ & $6.08 \%$ & $-5.2 \%$ \\
\hline Compras & $1.99 \%$ & $3.78 \%$ & $1.79 \%$ \\
\hline Distribución en planta & $7.61 \%$ & $3.13 \%$ & $-4.48 \%$ \\
\hline Distribución & $3.11 \%$ & $2.21 \%$ & $-0.9 \%$ \\
\hline Previsión & $1.02 \%$ & $1.66 \%$ & $0.64 \%$ \\
\hline Localización & $1.07 \%$ & $1.38 \%$ & $0.31 \%$ \\
\hline Planificación de la capacidad & $2.09 \%$ & $1.01 \%$ & $-1.08 \%$ \\
\hline Mantenimiento & $2.04 \%$ & $0.92 \%$ & $1.12 \%$ \\
\hline Dirección de proyectos & $1.43 \%$ & $0.64 \%$ & $-0.79 \%$ \\
\hline Planificación agregada & $0.66 \%$ & $0,55 \%$ & $-0,11 \%$ \\
\hline Medición del trabajo & $0.51 \%$ & $0.18 \%$ & $-0.33 \%$ \\
\hline Calidad en el trabajo & $0.20 \%$ & $0.09 \%$ & $-0.11 \%$ \\
\hline TOTAL & $100.00 \%$ & $100.00 \%$ & \\
\hline
\end{tabular}

Gestionar más allá de los límites de la empresa individual: Una oportunidad para obtener ventajas competitivas

Tabla 2. Temas de investigación de DO Abordados en los 90

Por tanto, durante los 80 los temas relacionados con la GCS no ocuparon una posición destacada en la investigación en DO. Sin embargo, los propios cambios que estaban experimentando las empresas y su entorno hacen que éstas, a principios de los 90 , consideren tanto a la función de compras como a la de distribución como áreas de gran interés (Malhotra et al, 1994) ${ }^{7}$. Ello no se corresponde con el propio desarrollo investigador en estos temas. En este sentido, si examinamos los trabajos de Filippini (1997) ${ }^{8}$ y Pannirselvam et al. $(1999)^{9}$, tanto los artículos en revistas como los trabajos presentados en congresos relativos a compras o distribución alcanzaban porcentajes incluso algo inferiores a los detectados a finales de los 80 (Tabla 2). Igualmente, al igual que ya ocurriera durante los 80 , programación de operaciones y control de inventarios seguían siendo los dos temas más abordados en los artículos de las

7 En este estudio se encuestan a los Vicepresidentes de Producción/Operaciones de 75 grandes compañías. 8 Analiza los 244 trabajos presentados en 1996 al congreso del Decision Science Institute (DSI). 
revistas de DO. No obstante, estos datos deben ser matizados debido a que la GCS todavía no estaba consolida y, por tanto, no se constituía como un área especializada y específica en DO en base a la cual clasificar los artículos. Así, los trabajos sobre GCS se colocaban en la categoría más próxima a su contenido (por ejemplo, estrategia, distribución o compras), como ocurre en el trabajo de Pannirselvam et al. (1999). Es por ello que estos autores, entre sus conclusiones, definen a la GCS como un campo emergente en la DO que, en futuros estudios bibliométricos, necesita ser analizado como una categoría separada del resto para conocer realmente su situación y evolución (al igual que proponen para los temas de desarrollo de nuevos productos, dirección de la tecnología o gestión medioambiental). En consecuencia, a finales de los 90 se pone de manifiesto la importancia de la GCS y la necesidad de investigaciones al respecto, pues éstas últimas todavía no han respondido a la creciente demanda que surge del ámbito empresarial. Así, hay autores (Handfield y Melnyk, 1998) que sitúan en esos momentos a la GCS como una de las áreas en la primera etapa (descubrimiento y descripción) del proceso de investigación y construcción de teorías.

Si nos centramos en una parcela más concreta dentro de la DO encontramos artículos que pueden ayudarnos a definir con mayor precisión la situación actual de la investigación en GCS. A este respecto, Prasad et al. (2000) analizan 587 artículos dedicados a Dirección de Operaciones Internacional obtenidos del estudio del total de artículos publicados en 24 revistas de DO en el período 1986-97. Sus resultados muestran un claro aumento de publicaciones dedicadas a cuatro aspectos, en concreto, distribución, estrategia de operaciones, aprovisionamiento y localización. Esto parece responder a la propia problemática que está experimentando la empresa respecto al proceso de globalización en el que se encuentra inmersa y que afecta profundamente a su cadena de suministro. Generalmente, las cadenas de suministro internacionales implican tiempos de suministro más largos, peores previsiones de ventas y mayores dificultades para responder a cambios en los pedidos, resultando a menudo mucho más largas y más complejas que las locales (Flaherty, 1996). Muy probablemente, si los autores hubieran definido una categoría para la GCS propiamente dicha, como proponían Pannirselvam et al. (1999), buena parte de los trabajos clasificados en las tres primeras categorías se desplazarían hacia la misma.

Esta suposición se corrobora en el trabajo de Alfaro et al. (2002) que analiza los artículos dirigidos específicamente a la GCS publicados en las 12 principales revistas de DO durante el período 1995-2001. Los artículos orientados a la GCS fueron un total de 376. Al analizar la evolución cronológica observaron un claro aumento en los últimos años de las publicaciones sobre GCS. Así, en 1995, como media, sólo el 2,29\% de los artículos de las revistas analizadas se centraban en este tema. Sin embargo, este porcentaje se situaba en el $7 \%$ en 2001. No obstante, se encuentra mucha diferencia entre las diversas revistas. Por ejemplo, la International Journal of Operations and Production Management pasó de un 5,88\% de publicaciones sobre GCS en 1995 a un 22,09\% en 2001. Además, este estudio también analizó los aspectos concretos de GCS tratados en los artículos, determinándose ,que el tema más investigado era el relativo al diseño de estrategias y modelos de GCS, abordados, preferentemente, mediante el estudio de casos. Ello es lógico si tenemos en cuenta que nos encontramos en una etapa inicial de investigación y creación de teorías en este campo.

Este auge de la investigación en GCS desde finales de los 90 se corrobora en trabajos posteriores (Rungtusanatham et al., 2003; Slack et al., 2004; Burgess et al., 2006). Rungtusanatham et al. (2003) realizan un estudio de 285 artículos empíricos publicados entre 1980-2000 en seis de las principales revistas de DO, identificando cinco temas básicos: JIT, Gestión de la Tecnología, Estrategia de Operaciones, Calidad y GCS. Aunque el número de publicaciones sobre GCS fue inferior al resto de categorías, la distribución temporal muestra que su crecimiento en los últimos 5 años fue el más elevado junto a las investigaciones en

9 Estos autores analizan 1754 artículos publicados entre 1992 y 1997 en las siete revistas más prestigiosas de DO, así como las actas de las conferencias del Decision Science Institute (DSI) y la Production and Operations Management Society (POMS) de los años 1996 y 1997. 
Estrategia de Operaciones. Burgess et al. (2006) analizan una muestra aleatoria de 100 artículos del período 1985-2003 obtenida en ABI/Inform Global Proquest con la búsqueda de "supply chain management" como palabra clave. El 77\% de los artículos obtenidos correspondían a los últimos años del período analizado (1999-2003). Slack et al. (2004) analizan los artículos publicados en el Journal of Operations Management y el International Journal of Operations and Production Management en el periodo 2000-2003. Los resultados muestran que casi el 15\% de los artículos se centraban en GCS, siendo solo superados por los publicados sobre Estrategia de Operaciones (20,25\%). Por tanto, la GCS muestra un crecimiento de la investigación exponencial en los últimos años.

Por su parte, el estudio de las actas de congresos consolida los datos anteriores obtenidos del análisis de las revistas. Así, Alfaro et al. (2001) determinaron el porcentaje de aportaciones sobre GCS que se habían presentado a los dos congresos más importantes de DO, como son el de la European Operations Management Association (EurOMA) y el de la Production and Operations Management Society (POMS). Los datos revelan un crecimiento muy significativo de los trabajos dedicados a la GCS, llegando a alcanzar el 21,48\% en el congreso de POMS celebrado en 2001 (Tabla 3). Esta tendencia se corrobora al analizar las actas de los últimos congresos de POMS y EurOMA, en los que la GCS comprende una parte sustancial de las sesiones. Por tanto, si tenemos en cuenta que las aportaciones a congresos son una muestra de la investigación del momento y un avance de las publicaciones en revistas especializadas, podemos afirmar que la GCS se consolida en la actualidad como uno de los temas de investigación más tratados en DO.

\begin{tabular}{ll}
\hline Congresos & Aportaciones sobre GCS (\%) \\
\hline EurOMA (1996) & $8.57 \%$ \\
EurOMA (1997) & $8.08 \%$ \\
POMS (1998) & $8.10 \%$ \\
EurOMA (1998) & $11.46 \%$ \\
POMS (1999) & $11.17 \%$ \\
EurOMA(1999) & $38.33 \%$ \\
POMS (2000) & $17.57 \%$ \\
POMS (2001) & $21.48 \%$ \\
\hline
\end{tabular}

Tabla 3. Porcentaje de aportaciones a congresos referentes a GCS (Fuente: Alfaro et al., 2001)

Si nos centramos, a continuación, en el contexto educativo, la incorporación a la formación de los nuevos conceptos relativos a la GCS muestra una tendencia más lenta que la propia actividad investigadora. Así, se observa que los manuales de DO existentes a mediados de los 90 (por ejemplo: Adam y Ebert, 1991; Heizer y Render, 1991; Chase y Aquilano, 1994; Schroeder, 1993; Domínguez Machuca et al., 1995a y 1995b; Fernández Sánchez, 1993) no dedicaban ningún capítulo específico a este tema. A este respecto, en un estudio de Parson y Globerson (1987), que analiza el contenido de los catorce principales manuales publicados entre 1984 y 1987, se detecta que sólo aparece, como concepto vinculado a la GCS, las cuestiones relativas a logística. Los manuales de este período orientaban la formación preferentemente hacia cuestiones operativas como la gestión del inventario y el empleo de técnicas como la programación lineal.

10 No obstante, el contenido que presentan los diferentes capítulos referidos a la GCS muestra gran diversidad en los aspectos abordados y la orientación de los mismos. Ello es debido a que estamos ante un campo emergente que todavía requiere de investigaciones que generen un cuerpo de conocimiento sólido respecto al tema tratado.

Gestionar más allá de los límites de la empresa individual: Una oportunidad para obtener ventajas

competitivas 
No será hasta finales de los 90 cuando los libros de texto de DO incorporen capítulos dedicados específicamente a la GCS (por ejemplo: Gaither y Fraizer, 1999; Rusell y Taylor, 1999; Heizer y Render, 2001; Krajewski y Ritzman, 2000) ${ }^{10}$. La tendencia ha ido más allá con la publicación de manuales que orientan la formación en DO dentro del nuevo enfoque de la GCS. En este sentido, entre los primeros manuales con este enfoque se encontraron los de Waller (2003) -Operations Management. A supply chain approach- o Vollmann et al. (2005) -Manufacturing Planning and Control for Supply Chain Management. Por tanto, la formación en DO se está orientando hacia una ruptura de las barreras estrictamente empresariales para situarlas en unos límites más amplios, como son los de la cadena de suministro.

En lo que se refiere a la incorporación efectiva de la GCS en los programas docentes, no existen muchas investigaciones al respecto. En EE.UU. la reacción ante este nuevo reto formativo, aunque sólida, se muestra algo tardía. Así, antes de 1995 muy pocas facultades de empresariales o ingeniería tenían cursos dedicados a la GCS, aunque se podían detectar conceptos relacionados con la cadena de suministro en asignaturas de Logística o de Dirección de Operaciones. Sin embargo, a partir del 2000 casi todas las titulaciones de Administración de Empresas y de Ingeniería más prestigiosas dedican, al menos, una asignatura a este tema, e incluso muchas la han incorporado al "core curricula" (Johnson y Pyke, 2000). No obstante, en los programas de las diferentes universidades se aprecia una amplia diversidad en los temas tratados y una gran variedad en la metodología docente empleada (Johnson y Pyke, 2000). Por su parte, en la universidad española el proceso se muestra más lento. Así, en los primeros años de este siglo, podemos observar que en las titulaciones de Administración y Dirección de de Empresas (Licenciaturas y Diplomaturas) así como de Ingeniería Industrial (tanto Técnicas como Superiores), no encontramos asignaturas dirigidas a la Gestión de la Cadena de Suministro (Machuca y Alfalla-Luque, 2002). No obstante, la GCS se ha podido incorporar no como una asignatura independiente, sino como un tema dentro de los programas de DO. Sin embargo, al analizar el contenido de 190 asignaturas de DO de 92 titulaciones de universidades españolas, se pudo comprobar que sólo se detectaron temas relacionados con la GCS en un escaso porcentaje (Machuca y Alfalla-Luque, 2002). En concreto, Logística aparecía en el 5,8\% de los programas y Gestión del Aprovisionamiento en el $3,6 \%$, no situándose en ninguna de las titulaciones analizadas dentro del núcleo básico de temas impartidos habitualmente en las asignaturas de DO.

En programas MBA sí parece darse una mayor importancia a este tema, incorporándose en el $80 \%$ de los master de las principales escuelas de negocios europeas desde finales de los 90 (Goffin, 1998). Por tanto, será en los primeros años de esta centuria cuando las universidades comiencen a responder a esta emergente necesidad formativa e introduzcan asignaturas de GCS en titulaciones de empresa, ingeniería y programas master (Johnson y Pyke, 2000; Larson y Halldorsson, 2002; Russell, 2007).

En conclusión, según los resultados analizados en este apartado, la GCS puede considerarse como uno de los temas de investigación más importantes en los inicios de este siglo (St. John et al., 2001) y una materia que es necesario incluir en la formación de los futuros directivos. Sin embargo, el desarrollo docente e investigador no parece evolucionar al mismo ritmo que las necesidades empresariales en este campo. 


\section{CONSIDERACIONES FINALES}

En los últimos años ha estado creciendo el consenso respecto a la importancia estratégica de la integración de proveedores, productores y clientes (Burt y Doyle, 1992; Clinton y Closs, 1997). El limitado concepto de logística ha ampliado sus fronteras expandiéndose por toda la cadena de suministro desde el proveedor de materias primas hasta el cliente final (Shapiro et al., 1993). Para los directivos, la importancia estratégica de la interconexión de proveedores, productores y clientes dentro de la cadena de suministro resulta cada día más evidente (Frohlich y Westbrook, 2001).

La Gestión de la Cadena de Suministro parte del propio reconocimiento de la complejidad de dichas cadenas. Esta complejidad es, en buena parte, fruto de la necesidad de establecer relaciones fluidas con los diferentes integrantes de la misma. Por ello, en la búsqueda de un funcionamiento eficiente, será necesario que los diferentes miembros trabajen en la consecución de un comportamiento integrado, compartan información, riesgos, costes y recompensas, cooperen hacia la búsqueda de una misma meta y un mismo objetivo de servicio al cliente, integren sus procesos y construyan y mantengan relaciones a largo plazo con los principales socios (Mentzer et al., 2001). Ello implicará, como hemos señalado, acciones en el largo, medio y corto plazo.

Sin embargo, la GCS no es una tarea fácil. Mentzer et al. (2001) diferencian lo que sería la "Orientación hacia la Cadena de Suministro" de la "Gestión de la Cadena de Suministro". La Orientación hacia la Cadena de Suministro se define como el reconocimiento por una organización de la cadena de suministro y de las implicaciones estratégicas de las actividades tácticas relacionadas con la dirección de los flujos en la misma. Una empresa tendrá una Orientación hacia la Cadena de Suministro si puede ver las implicaciones de dirigir sus flujos tanto aguas arriba como aguas abajo. Pero tener una Orientación hacia la Cadena de Suministro no implica poder implementarla, pues ello requiere una Orientación hacia la Cadena de Suministro de los diferentes eslabones que la integran. Una empresa puede implementar individualmente prácticas de cadena de suministro (entregas JIT, EDI con proveedores o clientes,...) pero esto no es GCS a menos que exista una coordinación (orientación estratégica) entre los eslabones. Por tanto, las compañías que quieran implementar la GCS deben tener primero una Orientación hacia la Cadena de Suministro. En otras palabras, la Orientación hacia la Cadena de Suministro es una filosofía y la GCS es la suma de todas las prácticas de dirección llevadas a cabo para implementar esta filosofía. Por tanto, una adecuada GCS nos debe llevar hacia una cadena de suministro integrada.

Aunque la GCS promueve ver el canal de suministro en su mayor amplitud, la realidad es que son relativamente pocas las empresas que han llegado a una completa integración de toda su cadena de suministro. Como se ha señalado, la mayoría de las empresas que practican la integración de la cadena de suministro limitan su alcance a un nivel hacia arriba y uno hacia abajo (Fawcett y Magan, 2002). Sin embargo, aquellas firmas que han tenido la capacidad de realizar una adecuada integración y gestión eficiente de su cadena de suministro están obteniendo claras ventajas competitivas. Por tanto, la GCS se presenta como un reto a afrontar tanto para las empresas como para los investigadores y docentes que, como se ha puesto de manifiesto, se han incorporado con cierto retraso en esta importante disciplina empresarial. Se demanda, en consecuencia, investigaciones que ayuden a la consolidación y mejora de este campo de estudio, así como una adecuada formación que prepare a los directivos (presentes y futuros) y les permita orientar su gestión para la búsqueda de ventajas competitivas a través de la GCS. 


\section{REFERENCIAS}

Adam, E.E. y Ebert, R.J. (1991): Administración de la Producción y las Operaciones. Prentice-Hall.

Volumen I

$\mathbf{N}^{\circ} \mathbf{2}$

2010
Alfalla-Luque, R. y Domínguez Machuca, J.A. (2002): La formación en Dirección de Producción/ Operaciones. El caso de la Universidad Española. Secretariado de Publicaciones de la Universidad de Sevilla.

Alfalla-Luque, R. y Machuca, J.A.D. (2000): El profesorado de Dirección y Gestión de Producción/ Operaciones en la Universidad española: Un estudio empírico. Cuadernos de Economía y Dirección de la Empresa, 6 (Mayo-Agosto), 286-316.

Alfalla-Luque, R. y Medina-Lopez, C. (2008): La cadena integrada, Logística Integral, Noviembre, 16-20.

Alfalla-Luque, R. y Medina-Lopez, C. (2009): Supply Chain Management: Unheard of in the 1970s, core to today's company. Business History, 51 (2), 201-220.

Alfaro Tanco, J.A., Álvarez Gil, M.J. y Montes, M.J. (2001): Gestión de la Cadena de Suministros: evolución y tratamiento en el ámbito de la Dirección de Operaciones. Actas XI Congreso Nacional de ACEDE.

Alfaro, J.A., Álvarez, M.J. y Montes, M.J. (2002): Lagging behind versus advancing too fast? Identifying Gaps Research in Supply Chain, Procedings of the $9^{\text {th }}$ International EurOMA Conference, 27-38.

Amoako-Gyampah, K. y Meredith, J.R. (1989): The Operations Management Research Agenda: An Update, Journal of Operations Management, 8 (3), 250-562.

Bagchi, P.K., Ha, B.C., Skjoett-Larsen, T. y Soerensen, L.B. (2005): Supply chain integration: a European survey. The International Journal of Logistic Management, 16 (2), 275-294.

Ballou, R.H. (1991): Logística Empresarial. Control y Planificación. Ed. Díaz de Santos, Madrid.

Bernabucci, R.J. (2008): Supply chain gains from integration, Financial Executive, 24 (3), 46-48.

Bessant, J., Kaplinsky, R. y Lamming, R. (2003): Putting supply chain learning into practice. International Journal of Operations and Production Management, 23 (2), 167-184.

Boyer, K.K., Frohlich, M. y Hult, G.T.M. (2005): Extending the Supply Chain. Ed. AMACOM, New York, NY. 
Briscoe, G. y Dainty, A. (2005): Construction supply chain integration: an elusive goal?, Supply Chain Management: An International Journal, 10 (4), 319-326.

Burgess, K., Singh, P.J., \& Koroglu, R. (2006). Supply chain management: A structured literature review and implications for future research. International Journal of Operations and Production Management, 26 (7), 703-729.

Burt, D.N. y Doyle, M.F. (1992): The american keiretsu, Business One. Irwin.

Cagliano, R., Caniato, F. y Spina, G. (2004): Lean, agile and traditional supply: how do they impact manufac-

Gestionar más allá de los límites de la empresa individual: Una oportunidad para obtener ventajas competitivas

Cagliano, R., Caniato, F. y Spina, G. (2006): The linkage between supply chain integration and manufacturing improvement programmes, International Journal of Operations \& Production Management, 26 (3), 282-299.

Chase, R.B. y Aquilano, N.J. (1994): Dirección y Administración de las Operaciones. Irwin.

Chase, R.B., Aquilano, N.J. y Jacobs, F.R. (2000): Administración de Producción y Operaciones. Irwin

Christopher, M. (1992): Logistics and supply chain management. London: Pitman Publishing.

Christopher, M. (2000): The agile supply chain. Competing in volatile markets, Industrial Marketing Management, 29 (1), 37-44.

Clinton, S.R. y Closs, D.J. (1997): Logistics strategy: does it exist? , Journal of Business Logistics, 18 (1), 1944.

Cooper, M.C. y Ellram, L.M. (1993): Characteristics of supply chain management and implications for purchasing and logistics strategy. The International Journal of Logistics Management, 4 (2), 13-22.

De Toni, A. y Nassimbeni, G. (1999): Buyer-supplier operational practices, sourcing policies and plant performance: results of an empirical research. International Journal of Production Research, 37 (3), 597619.

Díaz, A., Álvarez, M.J. y González, P. (2004): Logística inversa y medio ambiente. McGraw-Hill.

Elmuti, D. (2002): The perceived impact of supply chain management on organizational effectiveness, The Journal of Supply Chain Management, 49-57.

Fawcett, S.E. y Magan, G.M. (2002): The retoric and reality of Supply Chain Integration, International Journal of Physical Distribution \& Logistic Management, 32 (5), 339-361.

Fernández Sánchez, E. (1993): Dirección de la Producción I. Fundamentos Estratégicos. Cívitas. 
Fernández, E., Avella, L. y Fernández, M. (2003): Estrategia de Producción. McGraw-Hill.

Filippini, R. (1997): Operations management research: some reflections on evolution, models and empirical studies in OM, International Journal of Operations and Production Management, 17 (7), 655 -670 .

Volumen I

$\mathbf{N}^{\circ} \mathbf{2}$

2010

Fine, C.H. (1998): Clockspeed: Winning industry control in the age of temporary advantage. New York: Perseus Books.

Flaherty, M. T. (1996): Global Operations Management, McGraw-Hill.

Forza, C., Romano, P. y Vinelli, A. (2000): Information technology for managing the textile apparel chain. Current use, shortcomings and development directions. International Journal of Logistics: Research and Applications, 3 (3), 227-243.

Freeman, V. y Cavinato, J. (1990): Fitting Purchasing to the Strategic Firm: Frameworks, Processes, and Values. Journal of Purchasing and Materials Management, 26 (1), 19-25.

Frohlich M.T. y Westbrook R. (2001): Arcs of integration: an international study of supply chain strategies, Journal of Operations Management, 19, 185-200.

Gaither, N. y Frazier, G. (1999): Production and Operations Management. Thomson.

Gibson, B.J., Mentzer, J.T., \& Cook, R.L. (2005): Supply chain management: The pursuit of a consensus definition, Journal of Business Logistics, 26 (2), 17-25.

Gimenez, C. y Ventura, E. (2005): Logistics-production, logistics-marketing and external integration, their impact on performance. International Journal of Operations \& Production Management, 25 (1), 20-38.

Goffin, K. (1998): Operations Management Teaching on European MBA Programmes, International Journal of Operations and Production Management, 18 (5), 424-451.

Halldorsson, A., Kotzab, H., Mikkola, J. y Skjøtt-Larsen, T. (2007): Complementary Theories to Supply Chain Management. Supply Chain Management: An International Journal, 12 (4), 284-296

Hamblin, D. y Groves, G. (1995): Managing advanced manufacturing technology in the clothing industry. The Journal of Clothing Technology and Management, 12 (2), 1-12.

Handfield, R.B. y Nichols, E.L. (1999): Introduction to Supply Chain Management. Prentice-Hall, Upper Saddle River, NJ.

Handfield, R.B. y Nichols, E.L. (2002): Supply Chain Redesign - Transforming Supply Chains into Integrated Value Systems. Prentice-Hall, London.

Handfield, R.B. y Melnyk, S.A. (1998): The scientific theory-building process: a primer using the case of TQM, Journal of Operations Management, 16, 321-339.

Harrison, A. y Van Hoek, R. (2005): Logistics management and strategy. Pearson Education.

Heizer, J. y Render, B. (1991): Production and Operations Management: Strategies and Tactics. Allyn and Bacon.

Heizer, J. y Render, B. (2001): Dirección de la Producción. Decisiones Tácticas. Prentice Hall. 
Heizer, J. y Render, B. (2009): Principios de Administración de Operaciones. Prentice Hall.

Johnson, M.E. y Pyke, D.F (2000): Introduction to the special issue on teaching supply chain management, Production and Operations Management, 9 (1), 1-18.

Ketchen, D.J. y Guinipero, L. (2004): The intersection of strategic management and supply chain management. Industrial Marketing Management, 33,(1), 51-56.

Ketchen, D.J. y Hult, G.T.M. (2007): Bridging organization theory and supply chain management: The case of best value supply chains. Journal of Operations Management, 25, 573-580.

Kim, S.W. (2009): An investigation on the direct and indirect effect of supply chain integration on firm performance. Internatiional Journal of Production Economics, 119, 328-346.

Krajewski, L.J. y Ritzman, L..P. (2000): Administración de Operaciones; Estrategia y Análisis. Pearson Educación.

Kulp, S., Lee, H. y Ofek, E. (2004): Manufacturer benefits from information integration with retail customers. Management Science, 50 (4), 431-44.

Lambert, D. y Cooper, M. (2000): Issues in supply chain management. Industrial Marketing Management, 29(1), 65-83.

Lambert, D. M. (2001): Supply Chain Management: What does it involve?, Supply Chain \& Logistics Journal, Fall.

Lambert, D. M., Cooper, M.C. y Pagh, J.D. (1998): Supply chain management: implementation issues and research opportunities, The international journal of Logistics Management, 9, (2), 1-19.

Larson, P.D., \& A. Halldorsson. (2002). What is SCM? And, where is it? Journal of Supply Chain Management, 38 (4), 36-44.

Lee, H.L. (2000): Creating Value through Supply Chain Integration. Supply Chain Management Review, September, 1 .

Lummus, R.R. y Vokurka, R.J. (1999): Defining supply chain management: a historical perspective and practical guidelines, Industrial Management \& Data Systems, 99 (1), 11-17.

Machuca, J.A.D y Alfalla-Luque, R. (2002): Un análisis de los programas docentes de Dirección de Producción/Operaciones en la Universidad española: Un estudio empírico. Cuadernos de Economía y Dirección de la Empresa, 11, 149-185.

Machuca, J.A.D., Alvarez Gil, M.J., García González, S., Domínguez Machuca, M.A. y Ruiz Domínguez, A. (1995b): Dirección de Operaciones: aspectos tácticos y operativos en la producción y los servicios. McGraw-Hill.

Machuca, J.A.D., García González, S., Domínguez Machuca, M.A., Ruiz Jiménez, A. y Alvarez Gil, M.J. (1995a): Dirección de Operaciones: aspectos estratégicos en la producción y los servicios. McGraw-Hill. 
Malhotra, M.K., Steel, D.C. y Grover, V. (1994): Important Strategic and Tactical Manufacturing Issues in the 1990s", Decision Sciences, 25 (2), 189-214.

Manrodt, K.B., Holcomb, M.C. y Thompson, R.H. (1997): What's Missing in Supply Chain Management?. Supply Chain Management Review, September, 1.

Volumen I

$\mathbf{N}^{\circ} \mathbf{2}$

2010
Mejías Sacaluga, A., García Arca, J. y Prado Prado, J.C. (2001): Tecnología y Mejores Prácticas como factores facilitadores para la gestión de la Cadena de Suministro, Actas XI Congreso Nacional de ACEDE.

Mejza, M. y Wisner, J. (2001): The scope and span of supply chain management. International Journal of Logistics Management, 12 (2), 37-55.

Mentzer, J.T. (2004): Fundamentals of Supply Chain Management. Twelve Drivers of Competitive Advantage. Sage Publications, USA.

Mentzer, J.T., DeWitt, W., Keebler, J.S., Min, S. Nix, N.W. Smith, C.D, y Zacharia, Z.G. (2001): Defining supply chain management, Journal of Business Logistics, 22, (2), 1-25.

Meredith, J.R y Amoako-Gyampah, K. (1990): The Genealogy of Operations Management, Journal of Operations Management, 9 (2), 146-167.

Miller, J.G. y Graham, M.B.W. (1981): Production and Operations Management: Agenda for the '80s, Decision Sciences, 11, 547-571.

Miranda, F.J., Rubio, S., Chamorro, A. y Bañegil, T.M. (2005): Manual de Dirección de Operaciones. Thomson Learning.

Narasimhan, R. y Kim, S.W. (2002): Effect of supply chain integration on the relationship between diversification and performance: evidence from Japanese and Korean firms. Journal of Operations Management, 20, 303-323.

Pág 135

Oliver, R. y Webber, M. (1982): Supply Chain Management: Logistics catches up with Strategy. Outlook, 5, 42-47. [Reprint in Christopher (1992): Logistics: The Strategic Issue. London: Chapman and Hall, 63-75].

Pannirselvam, G.P., Ferguson, L.A., Ash, R.C. y Siferd, S.P. (1999): Operations management research: un update for the 1990's, Journal of Operations Management, 18, 95-112.

Parsons, R. y Globerson, S. (1987): Content Modification in Operations Management, Operations Management Review, 5 (3), 1-10.

Ponce, E. y Prida, B. (2004): La logística de aprovisionamientos para la integración de la cadena de suministros. Prentice Hall.

Porter, M.E. (1980): Competitive Strategy. Free Press. 
Prasad, S., Babbar, S. y Calis, A. (2000): International Operations Management and Operations Management Research: a comparative analysis, Omega. The International Journal of Management Science, 28, 97-110.

Quesada, G., Rachamadugu, R., Gonzalez, M. y Martinez, J.L. (2008). Linking order winning and external supply chain integration strategies. Supply chain management: An international journal, 13 (4), 296303.

Quinn, F. L. (1997): Team up for supply-chain success, Logistics Management, 16 (10), 39-41.

Ragatz, G.L., Handfield, R.B. y Scannell, T.V. (1997): Success factors for integrating suppliers into new prod-

Gestionar más allá de los límites de la empresa individual: Una oportunidad para obtener ventajas competitivas

Romano, P. (2003): Co-ordination and integration mechanisms to manage logistics processes across supply networks. Journal of Purchasing \& Supply Management, 9, 119-134.

Rosenzweig, E.D., Roth, A.V. y Dean, J.W. (2003): The influence of an integration strategy on competitive capabilities and business performance: an exploratory study of consumer products manufacturers. Journal of Operations Management, 21, 437-56.

Rungtusanatham, M.J., Choi, T.Y., Hollingworth, D.G., Wu, Z., \& Forza, C. (2003). Survey research in operations management: Historical analyses. Journal of Operations Management, 21 (4), 475-488.

Russell, S.H. (2007): Supply chain management: More than integrated logistics. Air Force Journal Logistics, XXXI, 2, 55-63.

Russell, R.S., Taylor, B.W. (1999): Operations Management: Focusing on Quality and Competitiveness. Prentice Hall.

Schroeder, R.G. (1993): Administración de Operaciones. McGraw-Hill.

Schroeder, R.G. (2000): Operations Management; Contemporary Concepts and Cases. McGraw-Hill.

Sezen, B. (2008): Relative effects of design, integration and information sharing on supply chain performance. Supply Chain Management: An International Journal, 13, (3), 233-240.

Shapiro, J.F., Singhal, V.M. y Wagner, S.N. (1993): Optimizing the value chain, Interfaces, 23, (2), 102-117.

Simchi-Levi, D., Kaminsky, P. y Simchi-Levi, E. (2000): Designing and Managing the Supply Chain. Concepts, Strategies, and Case Studies. Irwin McGraw-Hill, New York.

Slack, N., Lewis, M., \& Bates, H. (2004): The two worlds of operations management research and practice: Can they meet, should they meet? International Journal of Operations and Production Management, 24 (4), 372-387. 
Sprague, L.G. (2007): "Evolution of the field of operations management". Journal of Operations Management, 25, 219-238.

St. John, C.H., Cannon, A.R. y Pouder, R.W. (2001): “Change Drivers in the New Millennium: Implications for Manufacturing Strategy Research", Journal of Operations Management, 19, 143-160.

Volumen I Stevens, G.C. (1989): "Integrating the Supply Chain”, International Journal of Physical Distribution \& Materials Management, 19 (8), 3-8.

2010

Stevens, G.C. (1989): "Integrating the Supply Chain". International Journal of Physical Distribution \& Materials Management, 19 (8), 3-8.

Tan, K.C., Kannan, V.R. y Handfield, R.B. (1998): "Supply chain management: Supplier performance and firm performance". International Journal of Purchasing and Materials Management, 34 (3), 2-9.

Van der Vaart, T. y van Donk, D.P. (2008): "A critical review of survey-based research in supply chain integration". International Journal of Production Economics, 1 (11), 42-55.

Vickery, S.K., Jayaram, J., Droge, C. y Calantone, R. (2003):"The effects of an integrative supply chain strategy on customer service and financial performance: an analysis of direct versus indirect relationships". Journal of Operations Management, 21 (5), 523-539.

Vollmann, T.E., Berry, W.L., Whybark, D.C. y Jacobs, F.R. (2005): Manufacturing Planning and Control for Supply Chain Management. McGraw-Hill.

Waller, D.L. (2003): Operations Management. A supply chain approach. Thomson.

Wong, C.Y. y Boon-itt, S. (2008): "The influence of institutional norms and environmental uncertainty on supply chain integration in the Thai automotive industry". International Journal of Production Economics, 115, 400- 410.

Wood, A. (1997): "Extending the supply chain: Strengthening links with IT". Chemical Week, 159 (25), 25-26. 
Esta página fue dejada en blanco a propósito. 